\title{
Type 2 diabetes mellitus and thyroid disease: a two-sided analysis
}

\author{
Sotak S, Felsoci M, Lazurova I \\ Ist Department of Internal Medicine, L. Pasteur University Hospital and Faculty of Medicine, \\ Safarikiensis University Kosice, Slovakia. stefan.sotak@unlp.sk
}

\section{ABSTRACT}

AIM OF THE STUDY: To assess the prevalence of thyroid diseases in patients with type 2 diabetes mellitus (T2DM) in comparison with normal population; to determine prevalence of T2DM in patients with thyroid diseases. MATERIALS AND METHODS: First group consisted of 60 patients with T2DM without previous history of thyroid disease. Second group consisted of 60 patients with thyroid disease without any previously known impairment of glucose metabolism. Control group (CG) included 100 subjects who had no previous history of thyroid disease or glucose metabolism impairment. Blood tests were performed to evaluate thyroid and glucose metabolism parameters.

RESULTS: We found a significantly higher prevalence of thyroid diseases in patients with T2DM when compared to CG. Patients with T2DM showed to have higher serum levels of free triiodothyronine $\left(\mathrm{fT}_{3}\right)$, thyroid-stimulating hormone (TSH) and anti-thyroid peroxidase (anti-TPO) autoantibodies. We found no statistical significance in prevalence of T2DM in patients with thyroid diseases and CG. Among parameters of glucose metabolism, there were only higher fasting glucose levels in patients with hyperthyroidism and autoimmune thyroid disease (AITD). CONCLUSIONS: Patients with T2DM showed to have higher prevalence of AITD and primary hypothyroidism. We did not find higher prevalence of T2DM in patients with thyroid diseases (Tab. 3, Ref. 29). Text in PDF www.elis.sk. KEY WORDS: diabetes mellitus 2, thyroid disease.

\section{Introduction}

The association between thyroid diseases, especially though autoimmune diseases, and type 1 diabetes mellitus (T1DM) is well known. However, there is an increasing mass of data from recent years showing higher prevalence of thyroid diseases also in patients with T2DM $(1,2,3,4,5,6,7,8,9,10,11)$.

Low intake of iodine in diabetics with cardiovascular risk factors is responsible for higher prevalence of eufunctional goitre (EG), suppression of TSH induced by metformin, poor long-term glycemic control (11) and various humoral mechanisms like IGF-1 (10), nesfatin-1 (12) etc. The latter factors have been recognized as being most significantly participating in higher prevalence of thyroid diseases in T2DM patients.

On the other hand, a contribution of various forms of thyreopathy to the etiopathogenesis of T2DM has been demonstrated by several studies $(13,14)$. However, there are only poor data regarding the prevalence of T2DM in patients with thyroid diseases.

Ist Department of Internal Medicine, L. Pasteur University Hospital and Faculty of Medicine, Safarikiensis University Kosice, Slovakia

Address for correspondence: S. Sotak, Ist Department of Internal Medicine, L. Pasteur University Hospital and Faculty of Medicine, Safarikiensis University Kosice, Trieda SNP 1, SK-040 11 Kosice, Slovakia. Phone: +421.55 .6403420$

Acknowledgement: This research has been supported by the Scientific Agency of the Slovak Republic VEGA 1/0048/11
Independently on their etiology, both, hypo- and hyperthyroidism may affect glucoregulation in diabetics as well as in non-diabetics. The development of hyperinsulinism and insulin resistance together with thyroid hormones (TH)-induced hyperglycemia are typical for hyperthyroidism (14). On the other hand, insulin resistance as a result of hyperinsulinemia that leads to reduced glucose utilization in peripheral tissues $(15,16,17,18)$ is typical also for hypothyroidism $(18,19)$.

Several studies demonstrated a higher prevalence of AITD in both, type 1 and type 2 diabetics, when compared to the nondiabetic population (20). Although the exact cause of this association is still not known, there have been suggested genetic factors, i. e. deiodinase polymorphisms or influence of chronic infections that are more frequent in diabetics than in non-diabetics (21). Despite the fact that Hashimoto's thyroiditis in diabetics worsens the preexisting insulin resistance (22), it is still unclear whether the prevalence of T2DM in patients with euthyroid or hypothyroid AITD is increased.

The aim of this study was to assess the prevalence of thyroid diseases in patients with T2DM in comparison to a nondiabetic control group and to compare the basic thyroid parameters in both, diabetics and controls.

The second aim of this study was to examine the prevalence of T2DM or glucose metabolism impairment in patients with thyroid disorders and to assess the possible differences in parameters of carbohydrate metabolism in patients with various thyroid diseases. 


\section{Patients, materials and methods}

\section{Patients and materials}

This two-sided study included a total number of 120 patients and 100 controls that constituted the study population. Patients were divided into two groups.

The first group consisted of 60 patients with the established diagnosis of T2DM (31 male and 29 female) at average age 68.17 \pm 11.01 in range of $43-89$ years. All diabetic subjects were consecutively recruited from the $1^{\text {st }}$ Department of Internal Medicine of Louis Pasteur University Hospital in Košice, Slovakia and its outpatient office for diabetology from January 2013 to January 2015. Patients with previous history of thyroid disease or other endocrine disease were primarily excluded from the study.

The second group included 60 patients (10 male and 50 female) with thyroid disease at average age $68.7 \pm 13.4$ in range of 23-97 years who were also recruited from the $1^{\text {st }}$ Department of Internal Medicine of the Louis Pasteur University Hospital in Košice, Slovakia and its outpatient office for endocrinology during the same period of time. Thirty-seven of them ( 8 male and 29 female) of average age $68.35 \pm 12.21$, range $31-87$ years, suffered from primary hypothyroidism and 23 patients ( 1 male and 22 female) at average age $69.26 \pm 15.18$ in range of 23-97 years, have been diagnosed with hyperthyroidism (12 with Graves-Basedow disease, 1 with Hashitoxicosis, 6 patients with hypothyroidism on replacement therapy, actually overdosed, 3 with toxic thyroid adenoma and 1 with toxic multinodular goitre. Patients with previous history of prediabetes or diabetes were excluded from the study.

The control group consisted of 100 subjects ( 37 male and 63 female) at average age $63.85 \pm 18.98$ in range of 20-94 years. Diabetes mellitus, thyroid disease or any other endocrine diseases have been carefully excluded in all CG subjects .

The study was approved by the Ethics Committee of the Louis Pasteur University Hospital in Košice and written informed consent for the study was obligatory for all participants.

\section{Methods}

All patients and controls underwent clinical and laboratory evaluations. Basic anthropometric, thyroid and metabolic parameters as well as morphology of thyroid gland were assessed in every patient of both patient groups as well as in all CG subjects.

The following variables were assessed: age, gender, weight, body mass index (BMI), blood pressure and duration of T2DM or thyroid disease. Data on comorbidities such as hypertension, dyslipidemia and other symptoms of the metabolic syndrome were also obtained.

Fasting blood samples were collected from the patients and controls for laboratory assessment of $\mathrm{fT}_{3}$, free thyroxine ( $\mathrm{fT}_{4}$ ), and TSH serum levels as well as serum concentrations of autoantibodies to thyroglobulin (aTg) and aTPO. Additionally, fasting glycemia, serum C-peptide, insulin and glycosylated hemoglobin $\left(\mathrm{HbA}_{1 \mathrm{c}}\right)$ levels were assessed among parameters of glucose metabolism. Photometric colorimetry was used to examine glycemia and electrochemiluminescent immunochemistry to evaluate $\mathrm{C}$ peptide, insulin, $\mathrm{HbA}_{1 \mathrm{c}}, \mathrm{fT}_{3}, \mathrm{fT}_{4}, \mathrm{TSH}$, aTg and aTPO antibodies.
For the assessment of $\mathrm{HbA}_{1 \mathrm{c}}$, we used The Diabetes Control and Complications Trial (DCCT) standard with normal values $<6.5$ $\%$. BMI was calculated as weight $(\mathrm{kg})$ divided by the square of height $\left(\mathrm{m}^{2}\right)$. Homeostatic model assessment (HOMA) index was used to assess insulin sensitivity (glycemia x serum insulin / 22.5) while values $<2.4$ were considered normal.

Morphology and pathomorphology of thyroid gland were assessed using Esaote Technos MPx ultrasound and performed by the same ultrasonographer.

Diagnostic criteria for primary hypothyroidism included TSH serum concentrations $>4.2 \mathrm{mIU} / \mathrm{ml}$ and low or normal TH levels. Diagnostic criteria for primary hyperthyroidism included TSH serum concentrations $<0.27 \mathrm{mIU} / \mathrm{ml}$ and high or normal $\mathrm{TH}$ levels. Diagnostic criteria for AITD included positive ultrasonography (USG) image (diffuse homogenous or blotchy hypoechogenicity of thyroid tissue) and presence of aTg antibodies (serum levels above $115 \mathrm{kIU} / \mathrm{l}$ ) and/or aTPO antibodies (serum levels above $34 \mathrm{kIU} / \mathrm{l}$ ).

\section{Statistical analysis}

Continuous variables are presented as mean \pm standard error of mean (SEM); categorical variables are expressed as numbers or percentage of patients. Statistical significance of continuous variables comparison was assessed using the unpaired Student's t-test; comparison of categorical variable was conducted using Chi-square $\left(\chi^{2}\right)$ test with Yates correction. The software program of MS Excel 2003 and SPSS for Windows ver. 15.0 were used for statistical purpose. All statistical tests were two-tailed and p-value $<0.05$ was considered statistically significant.

\section{Results}

Mean values of particular anthropometric and thyroid parameters of patients with T2DM and their comparison with CG are shown in the Table 1. There was no difference in average mean age between T2DM and CG.

Tab. 1 Mean values of thyroid parameters in patients with T2DM and CG.

\begin{tabular}{lccc}
\hline & $\begin{array}{c}\text { T2DM } \\
(\mathrm{n}=60)\end{array}$ & $\begin{array}{c}\text { CG } \\
(\mathrm{n}=100)\end{array}$ & $\begin{array}{c}\text { Statistical } \\
\text { significance }\end{array}$ \\
\hline age (years) & $68.17 \pm 11.01$ & $63.85 \pm 18.98$ & $\mathrm{NS}$ \\
volume of thyroid gland (ml) & $15.05 \pm 4.47$ & $14.16 \pm 5.87$ & $\mathrm{NS}$ \\
fT3 (pmol/1) & $4.2 \pm 2.37$ & $3.27 \pm 0.88$ & $<0.01$ \\
fT4 (pmol/l) & $15.26 \pm 6.42$ & $16.57 \pm 4.65$ & $\mathrm{NS}$ \\
TSH (mIU/l) & $4.14 \pm 4.79$ & $1.95 \pm 1.63$ & $<0.01$ \\
aTPO (kIU/l) & $34.67 \pm 63.99$ & $12.77 \pm 21.65$ & $<0.01$ \\
aTg (kIU/l) & $61.28 \pm 153.64$ & $76.59 \pm 350$ & $\mathrm{NS}$ \\
\hline
\end{tabular}

Tab. 2. Prevalence of thyreopathies in patients with T2DM and CG.

\begin{tabular}{lccc}
\hline & $\begin{array}{c}\text { T2DM } \\
(\mathrm{n}=60)\end{array}$ & $\begin{array}{c}\mathrm{CG} \\
(\mathrm{n}=100)\end{array}$ & $\begin{array}{c}\text { Statistical } \\
\text { significance }\end{array}$ \\
\hline Any thyreopathy & $20(33.33 \%)$ & $18(18 \%)$ & $<0.05$ \\
hypothyroidism & $16(26.67 \%)$ & $5(5 \%)$ & $<0.01$ \\
hyperthyroidism & $1(1.67 \%)$ & $2(2 \%)$ & $\mathrm{NS}$ \\
AITD & $12(20 \%)$ & $8(8 \%)$ & $<0.05$ \\
\hline
\end{tabular}


Tab. 3. Mean values of glycid parameters in patients with thyreopathies and CG.

\begin{tabular}{|c|c|c|c|c|c|}
\hline & $\begin{array}{c}\text { Thyreopathies } \\
(\mathrm{n}=60)\end{array}$ & $\begin{array}{l}\text { Hyperthyroidism } \\
(\mathrm{n}=23)\end{array}$ & $\begin{array}{l}\text { Hypothyroidism } \\
(\mathrm{n}=37)\end{array}$ & $\begin{array}{l}\text { AITD } \\
(n=49)\end{array}$ & $\begin{array}{c}\text { CG } \\
(n=100)\end{array}$ \\
\hline Age (years) & $68.7 \pm 13.4$ & $69.26 \pm 15.18$ & $68.35 \pm 12.21$ & $69.18 \pm 13.14$ & $63.85 \pm 18.98$ \\
\hline BMI (kg/m2) & $26.12 \pm 4.95$ & $24.57 \pm 4.3$ & $27.08 \pm 5.06$ & $26.4 \pm 4.89$ & $25.04 \pm 4.83$ \\
\hline Fasting blood sugar $(\mathrm{mmol} / \mathrm{l})$ & $5.51 \pm 1.49 *$ & $5.95 \pm 2.18 * *$ & $5.23 \pm 0.71$ & $5.55 \pm 1.61^{*}$ & $5.08 \pm 0.99$ \\
\hline C-peptide $(\mu \mathrm{g} / \mathrm{l})$ & $3.56 \pm 1.69$ & $3.83 \pm 2.13$ & $3.4 \pm 1.34$ & $3.59 \pm 1.86$ & $3.73 \pm 2.21$ \\
\hline Insulin (mIU/l) & $13.19 \pm 6.81$ & $12.94 \pm 7.49$ & $13.35 \pm 6.37$ & $12.84 \pm 7.03$ & $11.5 \pm 9.6$ \\
\hline HOMA & $3.26 \pm 1.95$ & $3.5 \pm 2.43$ & $3.12 \pm 1.58$ & $3.19 \pm 2.02$ & $2.71 \pm 4.83$ \\
\hline $\mathrm{HbA} 1 \mathrm{C}(\%)$ & $5.46 \pm 0.64$ & $5.65 \pm 0.82$ & $5.34 \pm 0.46$ & $5.46 \pm 0.67$ & $5.28 \pm 0.94$ \\
\hline
\end{tabular}

$* \mathrm{p}<0.05, * * \mathrm{p}<0.01$

It has been shown that patients with T2DM have significantly higher serum TSH levels $(\mathrm{p}<0.01)$ and higher levels of aTPO antibodies than non-diabetic controls $(p<0.01)$ (Tab. 1). Furthermore, patients with T2DM had also slightly but significantly higher serum $\mathrm{fT}_{3}$ levels when compared with controls $(\mathrm{p}<0.01)$, while these values were within the normal range.

There was a significantly higher prevalence of hypothyroidism in patients with T2DM in comparison with controls (26.67 vs $5 \%$, respectively; $\mathrm{p}<0.01$ ) and a similar difference was observed also in the prevalence of AITD (20\% vs $8 \%$, respectively; $\mathrm{p}<$ 0.05) (Tab. 2).

The results from the second part of this study are presented in Table 3, demonstrating anthropometric and carbohydrate parameters in patients with thyroid diseases, hyperthyroidism, hypothyroidism and AITD and their comparison with subjects from CG. All groups were age- and BMI-matched. There were slightly but significantly higher fasting plasma glucose levels in patients with thyroid diseases when compared to subjects from CG (5.51 \pm 1.49 vs $5.08 \pm 0.99 \mathrm{mmol} / \mathrm{l}$, respectively; $\mathrm{p}<0.05$ ). However, significant difference between the groups was found neither in serum C-peptide, insulin and $\mathrm{HbA}_{1} \mathrm{c}$ levels, nor in HOMA index.

There were significantly higher fasting plasma glucose levels in the subgroup of patients with hyperthyroidism when matched with CG $(5.95 \pm 2.18 \mathrm{mmol} / 1$ vs $5.08 \pm 0.99 \mathrm{mmol} / 1$, respectively; $\mathrm{p}<0.01)$. No difference in this or any other metabolic parameters was observed in the subgroup with hypothyroidism, when compared to control subjects.

The prevalence of T2DM in the group of patients with thyroid disease was similar to that in CG (6.67 vs $4 \%$ ). Significant difference in the prevalence of T2DM was observed neither in patients with hypothyroidism nor in those with hyperthyroidism when compared to CG (2.7 vs $4 \%$ and 13.04 vs $4 \%$, respectively). We detected a slightly higher prevalence of T2DM in patients with AITD, however the difference did not reach the statistical significance $(8.16$ vs $4 \%$; $=0.07)$.

\section{Discussion}

In the first part of this study we demonstrated that the prevalence of AITD is significantly higher in patients with T2DM in comparison to nondiabetic controls. Among functional disorders, primary hypothyroidism was more common than hyperthyroidism and was more prevalent in diabetics than controls. This finding is in agreement with previous data $(3,4,6,8,11)$. Moreover, our patients with T2DM had significantly higher serum levels of TSH and aTPO antibodies when compared with controls.

In their study, Srindhi et al. (2013) demonstrated significantly higher serum levels of TSH in 75 patients with T2DM when compared to nondiabetic controls. However, in agreement with our study, they observe no difference in serum $\mathrm{fT}_{4}$ levels (7).

On the other hand, slightly higher serum TSH levels were observed also in 52 patients with type T2DM in a Japanese study of Moura et al (2013), albeit the difference from nondiabetic control group was not statistically significant. However, in contrast to our study the serum $\mathrm{fT}_{4}$ levels showed to be significantly higher, when compared to controls (5).

Our finding of serum $\mathrm{fT}_{3}$ levels being higher in diabetics than in controls is in conflict with the published data $(2,5,6,7,8,9$, 11). Previous studies did not confirm the differences in $\mathrm{fT}_{3}$ between T2DM and controls. While mean values of $\mathrm{fT}_{3}$ levels in both groups were in normal range, the most probable interpretation is only that of a mild but significant laboratory deviation. Secondly, higher levels of $\mathrm{fT}_{3}$ in diabetics might reflect a possible increased activity of type I deiodinase (DI), an enzyme which converts $\mathrm{fT}_{4}$ to $\mathrm{fT}_{3}$. However, the possibility of changes in DI activity in diabetic patients remains still unsolved (23).

Some studies also demonstrated a higher positivity of aTPO antibodies in diabetics which is in agreement with our study. Toulis et al (2014) described higher prevalence of AITD in 264 patients with T2DM than in a control nondiabetic group. In their study, the serum aTPO antibodies levels were higher in the diabetic group than in the nondiabetic one $(90 \pm 200$ vs. $60 \pm 156 \mathrm{kIU} / 1$, respectively; $p$ $<0.01$ ) (20). Contrary to the latter however, in the study of Díez and Iglesias (2014) the prevalence of autoimmune thyroid disease was lower in patients with T2DM than in healthy population (9).

Based on these controversial data, a routine screening of hypothyroidism in patients with T2DM still remains an unsolved question. It is well known that even subclinical hypothyroidism in patients with T2DM may increase the cardiovascular risk and contribute to an increase in the risk of microvascular complications such as diabetic nephropathy (24). From this aspect, and in respect of high prevalence of subclinical hypothyroidism in population, we suggest the routine screening of hypothyroidism to be reasonable $(7,25)$.

In the second part of our study, we demonstrated a significantly higher fasting glycemia in patients with hyperthyroidism 
when compared to healthy controls. Serum insulin levels as well as HOMA index were slightly but not significantly higher than these parameters in controls. It is well known that hyperthyroidism may lead to insulin resistance (14). Our results are partially in agreement with some previous studies (26).

The study of Al-Shoumer et al (2006) assessed glucose metabolism parameters of 30 patients with hyperthyroidism treated with carbimazol and propranolol and compared them with controls without thyroid disorder. Patients had significantly higher levels of fasting serum glucose, C-peptide and insulin. In these patients, they observed also marginally higher values of HOMA index (27).

The relationship between hypothyroidism and IR is attracting much more interest than hyperthyroidism. Kunal et al (2012) published a study of 8 patients with hypothyroidism without DM. When compared with their control euthyroid group, they showed to have significantly higher HOMA index and fasting insulin levels. On the other hand, fasting glycemia was not significantly increased (28). Although, the limiting point of this study was its small size, the findings of higher HOMA index, fasting insulin and fasting glycaemia levels were observed in another study as well (26). In our study, the prevalence of insulin resistance expressed by HOMA index in patients with hypothyroidism tended to be higher than in controls, albeit without significance. Similar results were achieved also by observations in patients with subclinical hypothyroidism. An Indian study on patients with subclinical hypothyroidism demonstrated higher values of HOMA index, as well as higher levels of fasting glycaemia and insulin (29). Although this study was similar to ours in size, patients were significantly younger than in our study.

According to our observation, patients with AITD had significantly higher fasting glucose levels than healthy controls. No differences in any other examined parameters were statistically significant. Actually, it is not possible to compare and draw conclusions from this finding in consideration of absence of large studies investigating the relationship between AITD and T2DM. Furthermore, none of patients with AITD in our group was in euthyroid state without the need of treatment. Therefore, we could investigate only the relationship between high or low thyroid function and glucose metabolism disorders, but we were not able to demark whether glucose metabolism disorders are mediated by thyroid malfunction or autoimmune inflammation alone. That is the main limitation of our study. Another limitation was that patients were in various stages of dysfunction because of treatment. Further studies on larger cohorts of especially untreated patients with euthyroid AITD are needed.

\section{Conclusion}

In summary, the prevalence of thyroid disease (AIT and primary hypothyroidism) in patients with T2DM is higher. We did not find the prevalence of T2DM to be higher in patients with any type of thyroid disease. Nevertheless, this is the first study assessing the relationship between T2DM and thyroid diseases from both sides and allows to recommend the thyroid function to be examined and screened for autoimmunity in patients with T2DM.

\section{References}

1. Xu M, Bi Y, Cui B, Hong J, Wang W, Ning G. The new perspectives on genetic studies of type 2 diabetes and thyroid diseases. Curr Genomics 2013 ; 14: 33-48.

2. Hage M, Zantout MS, Azar ST. Thyroid disorders and diabetes mellitus. J Thyroid Res (Internet). (cited 2015 Jul 31); 2011: 439463. www. ncbi.nlm.nih.gov/pmc/articles/PMC3139205/.

3. Al-Geffari M, Ahmad NA, Al-Sharqawi NA, Youssef AM, Al-Naqueb D, Al-Rubeaan K. Risk factors for thyroid dysfunction among type 2 diabetic patients in a highly diabetes mellitus prevalent society. Int J Endocrinol (Internet). (cited 2015 Jul 30); 2013: 417920. www.hindawi.com/ journals/ije/2013/417920/.

4. Kouidhi S, Berhouma R, Ammar M et al. Relationship of thyroid function with obesity and type 2 diabetes in euthyroid Tunisian subjects. Endocr Res 2013; 38: 15-23.

5. Moura NA, Parisi MC, Tambascia MA, Alegre SM, Pavin EJ, Yantut-Wittmann DE. The influence of body mass index and low-grade systemic inflammation on thyroid hormone abnormalities in patients with type 2 diabetes. Endocr J 2013; 60: 877-884.

6. Sousa Vergara Palma CCS, Pavesi M, Guedes Nogueira V et al. Prevalence of thyroid dysfunction in patients with diabetes mellitus. Diabetol Metab Syndr 2013; 5: 58 .

7. Srinidhi R, Kumar JA, Prajna K et al. Thyroid function in type 2 diabetes mellitus and in diabetic nephropathy. J Clin Diagn Res 2013; 7: $1583-1585$.

8. Basavaraj G, Niraj S, SidharthM. Study of thyroid profile in type 2 diabetes mellitus in comparison to non-diabetics. J Evol Med Dent Sci 2014; 3: 13684-13691.

9. Díez JJ, Iglesias P. An analysis of relative risk for goitre in euthyroid patients with type 2 diabetes. Clin Endocr (Oxford). 2014; 80: 356-361.

10. Duran AO, Anil C, Gursoy A et al. Thyroid volume in patients with glucose metabolism disorders. Arq Bras Endocrinol Metab 2014; 58 : 824-827.

11. Joffe BI, Distiller LA. Diabetes mellitus and hypothyroidism: strange bedfellows or mutual companions? World J Diabetes 2014; 5: 901-904.

12. Liu F, Yang Q, Gao N, Liu F, Chen S. Decreased plasma nesfatin-1 level is related to the thyroid dysfunction in pacients with type 2 diabetes mellitus. J Diab Res (Internet). (cited 2015 Jul 20); 2014: 128014. www. ncbi.nlm.nih.gov/pmc/articles/PMC4065684/.

13. Perros P, McCrimmon RJ, Shaw G, Frier BM. Frequency of thyroid dysfunction in diabetic patients: Value of annual screening. Diab Med. 1995; 12: 622-627.

14. Wartofsky L. Thyrotoxic storm. In: BRAVERMAN LE, UTIGER RD, editors. Werner and Ingbar's The Thyroid. Philadelphia: LippincottRaven; 2000, pp. 679-684.

15. Dimitriadis, G., Parry-Billings M, Bevan $\mathbf{S}$ et al. The effects of insulin on transport and metabolism of glucose in skeletal muscle from hyperthyroid and hypothyroid rats. Eur J Clin Invest 1997; 27: 475-483.

16. Rochon C, Taueron I, Dejax C et al. Response of glucose disposal to hyperinsulinaemia in human hypothyroidism and hyperthyroidism. Clin Sci 2003; 104: 7-15.

17. Cettour-Rose P, Samec S, Russell AP et al. Redistribution of glucose from skeletal muscle to adipose tissue during catch-up fat: a link between catch-up growth and later metabolic syndrome. Diabetes 2005; 54 (3): 751-756. 
18. Dimitriadis G, Mitrou P, Lambadiari V et al. Insulin action in adipose tissue and muscle in hypothyroidism. J Clin Endocrinol Metab 2006; 91 (12): 4930-4937.

19. Maratou E, Hadjidakis DJ, Kollias A et al. Studies of insulin resistance in patients with clinical and subclinical hypothyroidism. Eur J Endocrinol 2009; 160 (5): 785-790.

20. Toulis K, Tsekmekidou X, Potolidis $\mathbf{E}$ et al. Thyroid autoimmunity in the context of type 2 diabetes mellitus: Implications for vitamin D. Int J Endocrinol (Internet). (cited 2015 Jul 30); 2014: 710363. www.hindawi. com/journals/ije/2015/710363/.

21. Schroner Z, Lazúrová I, Petrovičová J. Autoimmune thyroid diseases in patients with diabetes mellitus. Bratis1 Lek Listy 2008; 109 (3): 125-129.

22. Gierach M, Gierach J, Skowrońska A et al. Hashimoto's thyroiditis and carbohydrate metabolism disorders in patients hospitalised in the Department of Endocrinology and Diabetology of Ludwik Rydygier Collegium Medicum in Bydgoszcz between 2001 and 2010. Endokrynol Pol 2012; 63 (1): 14-17.

23. Bianco AC, Kim BW. Deiodinases: implications of the local control of thyroid hormone action. J Clin Invest 2006; 116 (10): 2571-2579.
24. Chen HS, Wu TE, Jep TS et al. Subclinical hypothyroidism is a risk factor for nephropathy and cardiovascular diseases in type 2 diabetics patients. Diabet Med 2007; 24 (12): 1336-1344.

25. Furukawa S, Yamamoto S, Todo $Y$ et al. Association between subclinical hypothyroidism and diabetic nephropathy in patients with type 2 diabetes mellitus. Endocr J. 2014; 61: 1011-1018.

26. B UU, Mn S, Km S et al. Effect of insulin resistance in assessing the clinical outcome of clinical and subclinical hypothyroid patients. J Clin Diagn Res 2015; 9 (2): 1-4.

27. Al-Shoumer KAS, Vasanthy BA, Al-Zaid MM. Effect of treatment of hypertensidism on glucose homeostasis, insulin secretion, and markers of bone turnover. Endocr Pract 2006; 12 (2): 121-130.

28. Kunal KB, Parloop BA, Jigna SS. Association between altered thyroid state and insulin resistance. J Pharmacol Pharmacother 2012; 3 (2): $156-160$.

29. Vyakaranam S, Vanaparthy S, Nori S et al. Study of insulin resistance in subclinical hypothyroidism. Int J Health Sci Res 2014; 4 (9): 147-153.

Received January 13, 2018. Accepted February 27, 2018. 